\title{
DETECTION AND PARTIAL GENETIC CHARACTERISATION OF A NOVEL VARIANT OF AVIAN NEPHRITIS VIRUS IN INDIAN POULTRY FLOCKS SHOWING DIVERSE CLINICAL SIGNS
}

\author{
Vasudevan GowTHAMAN ${ }^{1}$, Sambu Dhayal SINGH ${ }^{1}$, Kuldeep DHAMA ${ }^{1}$, \\ Rajamani BARATHIDASAN ${ }^{1}$, Palani SRINIVASAN ${ }^{2}$, Sellappan SARAVANAN ${ }^{2}$, \\ Thippichettypalayam Ramasamy GOPALAKRISHNAMURTHY ${ }^{2}$, Rajib DEB $^{3}$, \\ Basavaraj MATHAPATI ${ }^{4}$ and Muthannan Andavar RAMAKRISHNAN ${ }^{4 *}$ \\ ${ }^{1}$ Avian Diseases Section, Division of Pathology, Indian Veterinary Research Institute, \\ Izatnagar, Uttar Pradesh - 243 122, India; ${ }^{2}$ Poultry Disease Diagnosis and Surveillance \\ Laboratory, Veterinary College and Research Institute Campus, Namakkal, Tamil Nadu, \\ India; ${ }^{3}$ Project Directorate on Cattle (ICAR), Meerut, Uttar Pradesh, India; ${ }^{4}$ Division of \\ Virology, Indian Veterinary Research Institute, Mukteshwar, Uttarakhand, India
}

(Received 18 December 2014; accepted 22 June 2015)

\begin{abstract}
Avian nephritis virus (ANV) infects poultry flocks worldwide, but no confirmed cases have been reported from India so far. In the current study, disease investigation was carried out in 21 broiler flocks at different parts of India with clinical signs of nephritis, uneven and stunted growth, diarrhoea, reduced body weight, and mortality up to $9.72 \%$. Out of the 21 flocks screened, two were found positive for ANV in RT-PCR assay. BLAST analysis revealed that the ANV of Indian origin was closely related to ANV-1 strains reported from Japan, Hungary and China. However, comparison of a small portion $(\sim 12 \%$ of nucleotides, i.e. $\sim 60$ nts, common site for ANV-1 and ANV-3, position 2200-2260 of ORF 1a gene) of the Indian ANV sequence with ANV-3 sequences revealed 89-93\% identities with different ANV-3 isolates. Phylogenetically, ANV-1 forms three clades, and the Indian ANV clustered under clade II. This study confirms the existence of ANV in Indian poultry flocks and is the first report on the molecular detection and genetic characterisation of ANV from India.
\end{abstract}

Key words: Avian nephritis virus, clade II, astrovirus, RT-PCR, phylogenetic analysis, ORF 1a gene

The family Astroviridae is divided into two genera, Mamastrovirus (MAstV) and Avastrovirus (AAstV) that consist of astroviruses of mammalian and avian species, respectively (Bosch et al., 2012). Previously, species in the Avastrovirus genus were defined on the basis of hosts from which they were isolated (e.g. Chicken astrovirus, Duck astrovirus, Turkey astrovirus, etc.). Since the species do not correspond to genetic phylogenies, based on genetic analysis

*Corresponding author; E-mail: maramakrishnan@gmail.com 
of the complete capsid region avian astroviruses of the genus Avastrovirus have recently been reclassified into three species, i.e. Avastrovirus 1 (including Turkey astrovirus 1), Avastrovirus 2 (including Avian nephritis virus 1 and 2), Avastrovirus 3 (including Turkey astrovirus 2 and Duck astrovirus) [Virus Taxonomy: 2014 Release; EC 46, Montreal, Canada, July 2014, E-mail ratification 2015 (MSL \#29); http://ictvonline.org/virustaxonomy.asp].

Avian nephritis virus 1 (ANV-1) had originally been isolated from a 1week-old healthy broiler chick (Yamaguchi et al., 1979). This virus causes mild growth depression, interstitial nephritis and sometimes even mortality, especially after infection of 1-day-old specific pathogen free (SPF) chickens. ANV-2 was reported by several Japanese groups (Takase et al., 1989; Shirai et al., 1992). Recently, a novel group of ANV has been found in chicken and turkey flocks with enteric and locomotion problems, and tentatively classified as ANV-3; this virus causes severe interstitial nephritis, runting-stunting syndrome-like (RSS) signs and even mortality after inoculation into 3-week-old SPF layer chickens (de Wit et al., 2011). The present study reports the first molecular detection and genetic analysis of ANV in Indian poultry flocks showing different clinical signs.

\section{Materials and methods}

\section{Disease investigation and sample collection}

A disease investigation was carried out in 21 broiler flocks from four different states of India (Uttar Pradesh, Karnataka, Andhra Pradesh, and Tamil Nadu) between May 2010 and August 2011. The flocks were aged 7-23 days and showed the incidence of nephritis with or without visceral gout, diarrhoea and stunted growth. Freshly dead birds were subjected to postmortem examination. Five birds per flock which showed severe lesions were selected for sampling. Tissue samples of trachea, lungs, proventriculus, kidney and spleen were pooled and used for molecular diagnosis. Kidney samples in $10 \%$ neutral formal saline were used for histopathological examination (Luna, 1968). Feed samples and tissue samples (liver and kidney) were collected and outsourced for mycotoxin analysis by thin-layer chromatography (TLC).

\section{Bacterial isolation}

The external surface of the heart was sterilised with a hot scalpel blade, an incision was made and blood swabbing was carried out. The heart blood swabs were processed according to standard methods for bacterial isolation (DufourZavala et al., 2008). One swab was streaked on 5\% sheep blood agar and another on MacConkey agar. 


\section{$R N A$ extraction and $R T-P C R$}

The tissue samples were homogenised in phosphate-buffered saline to a final concentration of $10 \%(\mathrm{w} / \mathrm{v})$ and centrifuged with $4,500 \mathrm{~g}$ for $15 \mathrm{~min}$. Viral RNA was extracted from the supernatant using QIAamp Viral RNA Mini Kit (Qiagen, Germany). cDNA was synthesised using SuperScript ${ }^{\circledR}$ First-Strand Synthesis System (Invitrogen, Germany) employing random primers. Amplification of the ORF 1a gene (GP1) of ANV was performed using the published primers (5'-AGATACGCTTGCTCGTCTTG-3') and (5'-CCTCTAACCGGCGATATTCT3') (Mándoki et al., 2006). The reaction was run using HotStar Taq ${ }^{\circledR}$ Master Mix (Qiagen, Germany) with an initial denaturation at $95^{\circ} \mathrm{C}$ for $15 \mathrm{~min}$, followed by 35 cycles at $95{ }^{\circ} \mathrm{C}$ for $1 \mathrm{~min}, 6{ }^{\circ} \mathrm{C}$ for $1 \mathrm{~min}, 72^{\circ} \mathrm{C}$ for $1 \mathrm{~min}$, and a final extension at $72{ }^{\circ} \mathrm{C}$ for $10 \mathrm{~min}$. The samples were also screened for the presence of avian reovirus (ARV), chicken anaemia virus (CAV), infectious bronchitis virus (IBV) and infectious bursal disease virus (IBDV) by PCR/RT-PCR using published primers (Ottiger, 2010).

\section{Sequencing and genetic analysis}

The RT-PCR amplified products were purified using ExoSAP-IT ${ }^{\circledR}$ (Affymetrix, USA) and sent for commercial sequencing. The nucleotide (nt) sequence of the PCR products was identified by BLAST, then compared with ANV sequences available in the NCBI GenBank by using the MEGA 6 programme. A phylogenetic tree was constructed in MEGA 6 using the Maximum Likelihood method based on the Tamura-Nei model with 1000 bootstrap replications (Tamura et al., 2013).

\section{Results}

Two out of 21 poultry flocks were found positive for ANV by RT-PCR. These two positive flocks were found negative for ARV, CAV, IBV, and IBDV. No mycotoxins (aflatoxin, ochratoxin, citrinin and T-2 toxin) could be detected in the tissue samples; however, the feed samples contained traces of aflatoxin ( $2 \mathrm{ppb}$ ). In the remaining 19 flocks, CAV, IBV, IBDV, and E. coli were detected either as single pathogens or in combination (data not shown).

Both ANV-positive flocks were from the State of Tamil Nadu, and the distance between the two farms is two kilometres. Both flocks were reared in deeplitter housing system, had free movement of vehicles and farm personnel, received chicks from the same hatchery, had a vaccination history against NDV at 4 days of age, and were treated with enrofloxacin at $10 \mathrm{mg} / \mathrm{kg}$ body weight. On the day of investigation, the age of the birds was 19 (Flock A) and 21 (Flock B) days. According to the history, the clinical disease started on day 6 in Flock A and day 8 in Flock B. Nearly $15-20 \%$ of the birds showed poor feed conversion 
ratio (FCR), stunted and uneven growth (Fig. 1), diarrhoea, staggering gait, and spread-out legs. On the day of culling (days 38 and 42, respectively), the overall mortality in Flock A and Flock B was 5.0\% and 9.72\%, respectively. Postmortem examination revealed pale swollen kidneys, thickening of the proventriculus wall, and visceral gout in the majority of the birds (Fig. 2). Microscopic examination of the kidneys revealed moderate to severe mononuclear infiltration in the interstitial space, tubular degeneration and necrosis (Fig. 3).

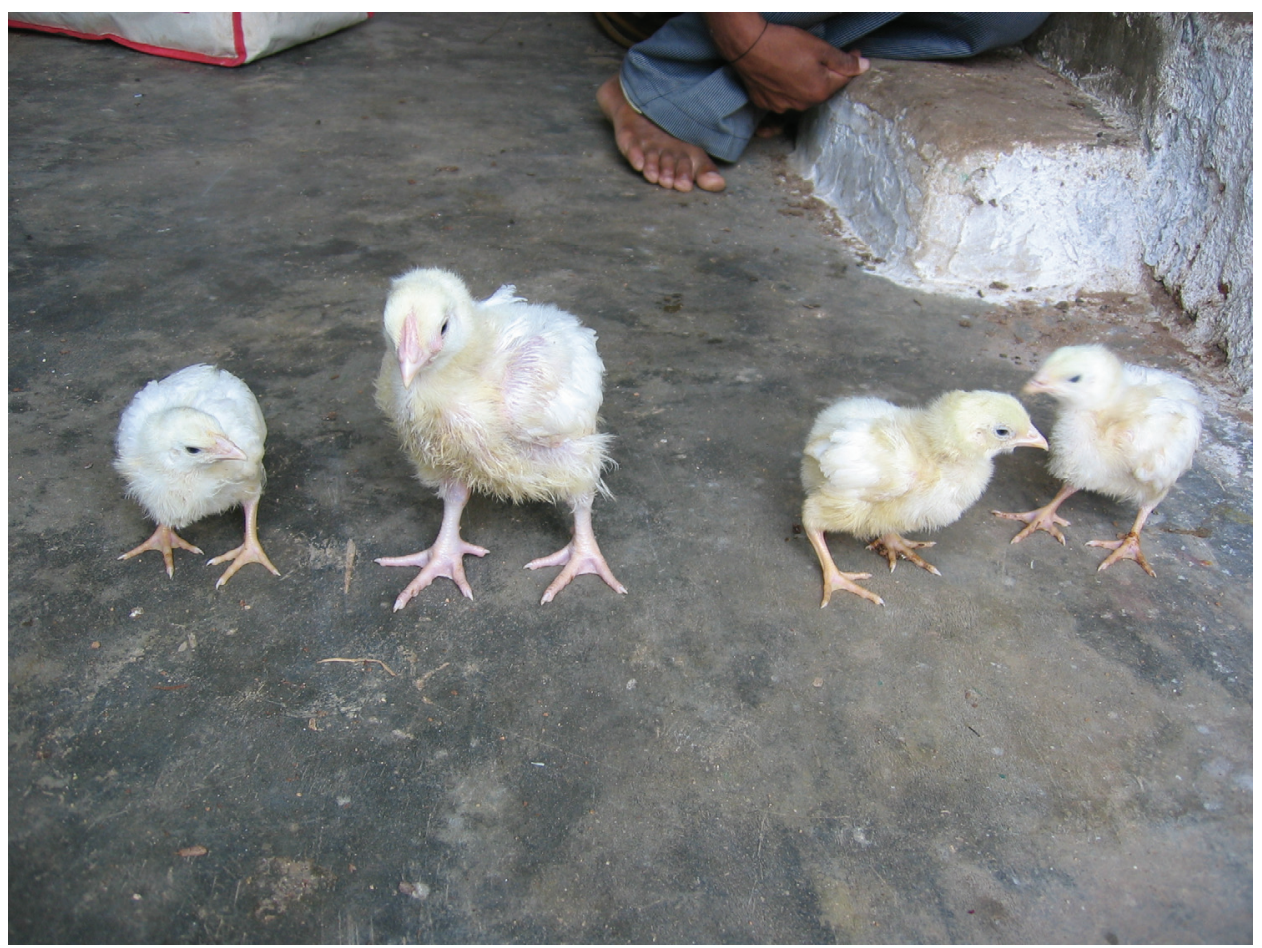

Fig. 1. The affected chickens exhibited runting-stunting syndrome-like signs

The nucleotide sequences (505 nt; position 1756-2260 of ORF 1 of G4260 Japanese ANV strain) of ANV from both flocks were $100 \%$ identical and, therefore, only one sequence was deposited in GenBank (KC411816.1). The genetic analysis revealed that the Indian ANV sequence was closely related to the ANV-1 sequences reported from Japan, Australia, China, and Hungary. It shared $87 \%$ nt identity with strain G-4260 (chicken - 1976) from Japan; 85\% nt identity with VIC-6a (chicken - 2010) and VIC-6b (chicken - 2010) from Australia, Sichuan (chicken - 2009), pigeon avian nephritis virus strain SH10 (pigeon 2010) of Shanghai, China; and $81-85 \%$ identities with several Hungarian isolates. Since complete sequences of ANV-3 are not available in GenBank, only a 
small portion (common site for ANV-1 and ANV-3; position 2200-2260 of ORF 1 of G-4260 Japanese ANV strain) of the Indian ANV sequence ( $12 \%$ of nucleotides, i.e. $\sim 60 \mathrm{nts}$ ) could be compared with available ANV-3 sequences; at this position, the Indian ANV shared 89-93\% identities with different ANV-3 isolates (data not shown). Phylogenetic analysis (449 nt; position 1756-2204 of ORF 1 of G-4260 Japanese ANV strain) revealed at least three major clades of ANV-1. The Indian ANV clustered with the Japanese (G-4260) and Australian sequences (Clade II) (Fig. 4).

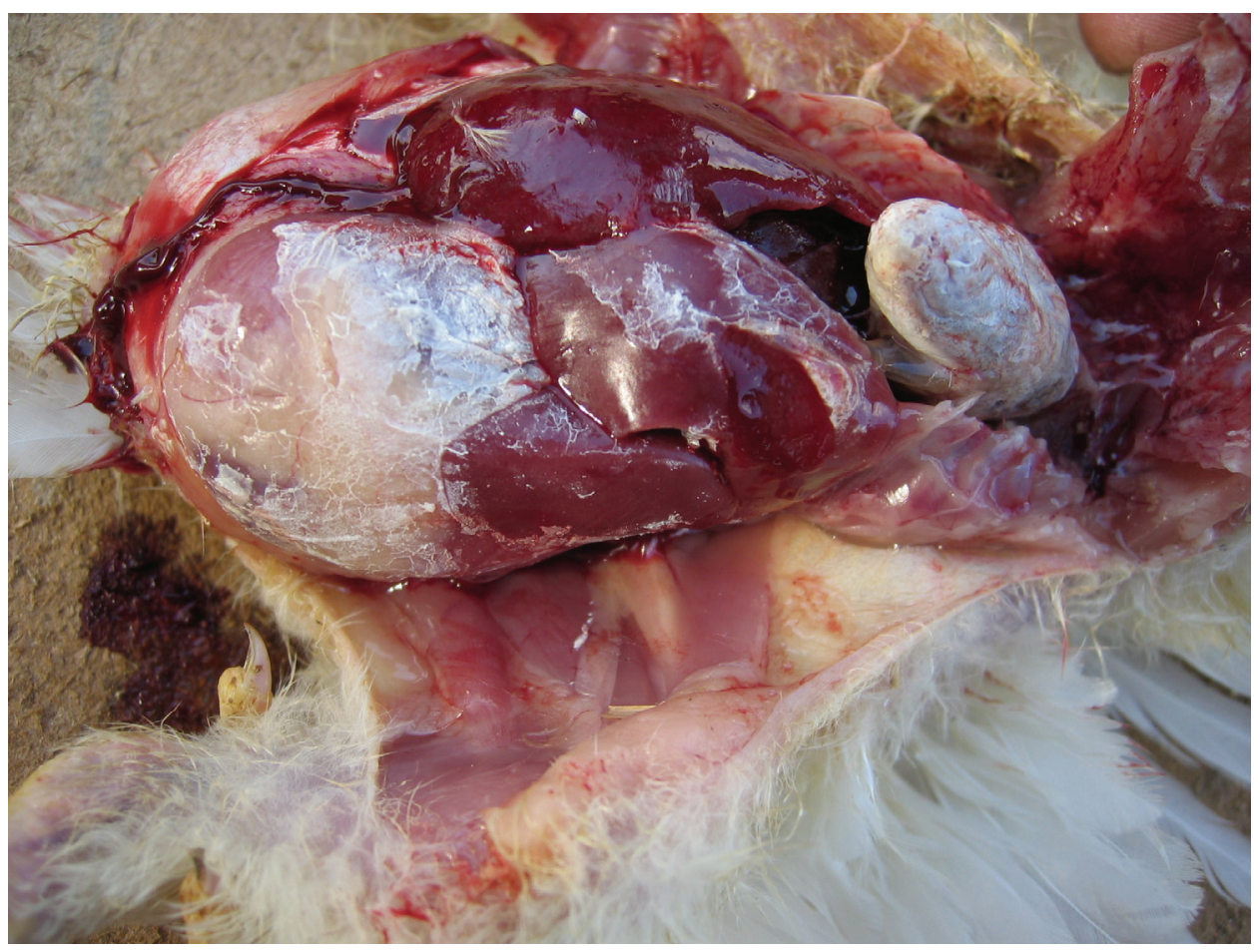

Fig. 2. Severe visceral gout in an affected chicken

\section{Discussion}

The present study describes diagnostic investigations performed in broiler farms showing incidence of nephritis and stunted growth. Avian nephritis viruses are difficult to isolate in cell culture due to their comparatively poor growth in primary chicken cell cultures. Generally, electron microscopy, immunofluorescence, and ELISA are used to diagnose astroviruses (Tang et al., 2005). For some years, direct RT-PCR has been successfully employed to more specifically 
detect ANV in field samples (Tang et al., 2005; Smyth et al., 2009; Hewson et al., 2010; Todd et al., 2010). Detection of ANV by RT-PCR and the absence of other viral aetiological agents and mycotoxins from the tissues collected from the two affected flocks indicated the possible involvement of ANV in RSS. In this case, RSS was more of a consequence of diarrhoea, and there were typical signs of nephritis as well.

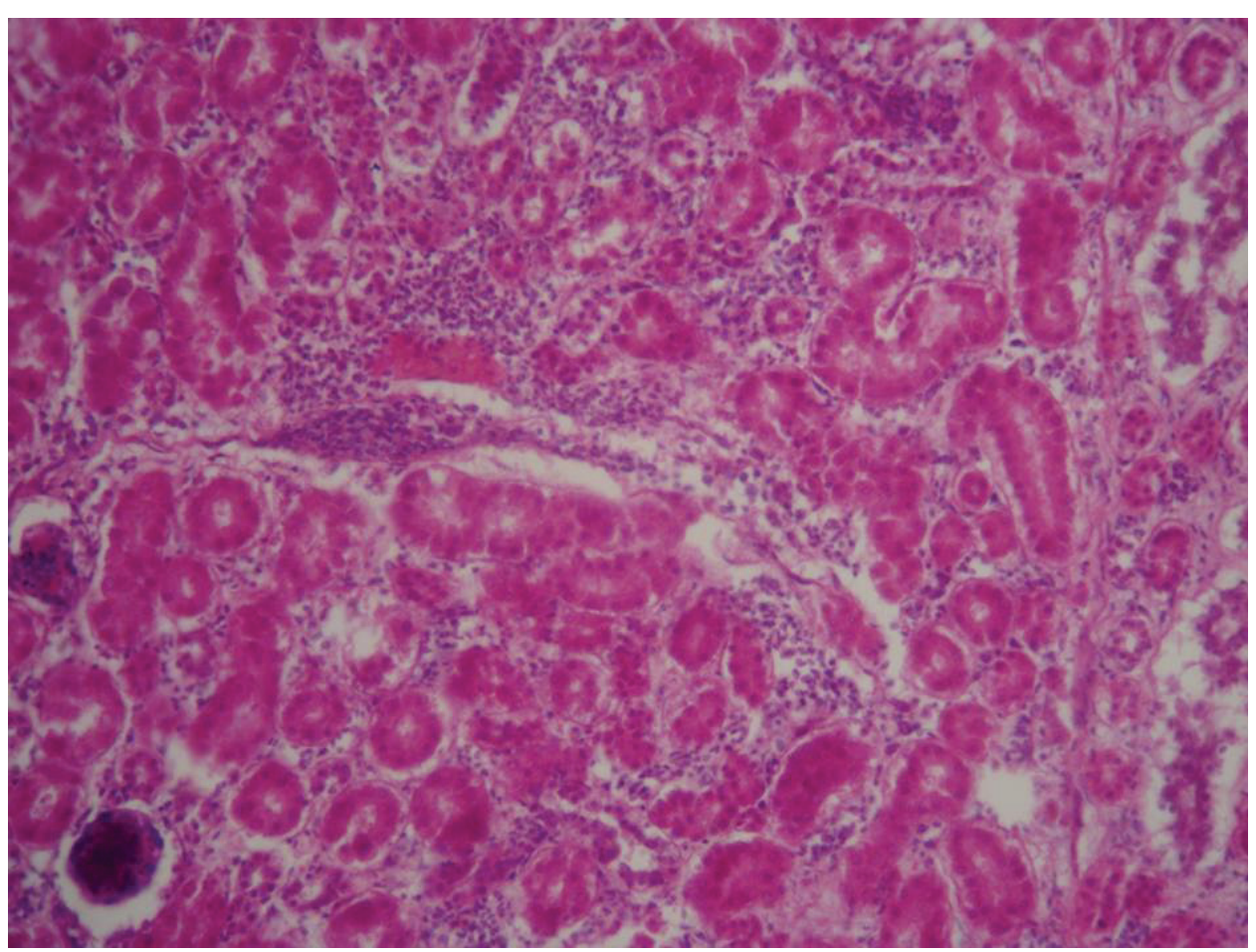

Fig. 3. Severe interstitial nephritis in a chicken infected by avian nephritis virus. Marked infiltration by mononuclear cells in the interstitial space of the renal tubules is apparent. Haematoxylin and eosin staining

The clinical findings observed in the present study are in agreement with those described in earlier reports (Shirai et al., 1991; Mándoki et al., 2006; de Wit et al., 2011). Under field conditions, clinical signs associated with astrovirus infection in broiler chicken have been known to vary from subclinical to outbreaks of RSS and baby chick nephropathy (Shirai et al., 1991; Pantin-Jackwood et al., 2011). Chickens of all ages may be infected with ANV but 1-day-old chicks are the most susceptible (Imada et al., 1982; Narita et al., 1990). Recently, a novel group of astrovirus termed ANV-3 was detected in samples from chicken and turkey flocks with clinical disorders such as tenosynovitis, enteric problems, 
or RSS-like conditions; experimentally, pathogenicity was observed after infection of 3-week-old SPF layers (de Wit et al., 2011). In our study, the affected flocks showed stunted and uneven growth, diarrhoea, staggering gait, and spread-out legs. ANV infection usually causes destruction of the renal tubules followed by reactive inflammation with mononuclear cell infiltration, called nephrosis-nephritis, leading to gout (uricosis) and finally the death of affected chickens. However, the majority of birds suffer from the subclinical form of the disease and grow slower than expected, causing economic losses to farmers (Shirai et al., 1989; Mándoki et al., 2006). The pathogenicity of ANV-1 is generally limited to the kidney of chickens, whereas ANV-3 damages the kidney, pancreas, duodenum, thymus, bursa, and liver (de Wit et al., 2011).

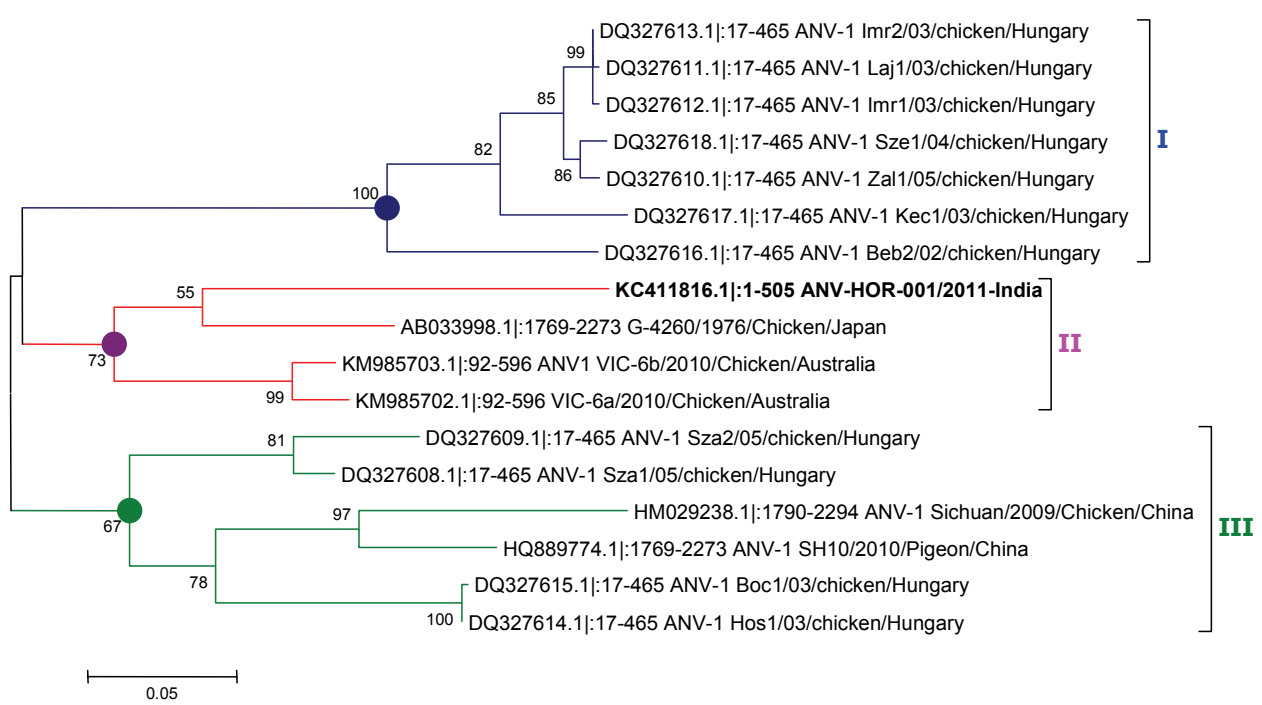

Fig. 4. Molecular phylogenetic analysis of avian nephritis virus. The evolutionary history was inferred by using the Maximum Likelihood method based on the Tamura-Nei model. A discrete gamma distribution was used to model evolutionary rate differences among sites. The analysis involved 17 nucleotide sequences. All positions containing gaps and missing data were eliminated. There were a total of 449 positions in the final dataset. Evolutionary analyses were conducted in MEGA6

Although it is generally believed that ANV infection produces nephritis only in 1-day-old chickens, in our study the viral nucleic acids could be detected in 19- to 21-day-old birds. This result is in agreement with the study of Mándoki et al. (2006), in which ANV nucleic acid could be detected in chicks up to 3 weeks of age. Genetic analysis of the Indian ANV has revealed that its closest relatives were ANV-1 isolates from Japan, Australia, China, and Hungary (81 to $87 \%$ nt sequence identity on a 505 -nt-long fragment from the ORF 1a corre- 
sponding to nt positions 1756 to 2260 in the ORF 1 of G-4260). Since the complete genome sequence of ANV-3 is not available and our PCR products were amplified from a somewhat different genome region, only a small portion of the ORF 1a sequence (positions 2200-2260) could be compared. This portion of the Indian ANV sequence had 89-93\% identities with different ANV-3 sequences. Although the Indian ANV sequence is closest (87\% nt identity) to strain G-4260 (chicken - 1976) from Japan, sequencing of common sites between ANV-1 and ANV-3 may identify the exact ANV type involved in the current study. Interestingly, phylogenetic analysis of ANV-1 based on the ML method revealed at least three clades within ANV-1. This finding may be helpful in molecular epidemiological studies of ANV-1. Evidence for recombination of ANV-1 and ANV-2 exists, and high nucleotide diversity is also known to occur in ANVs (Todd et al., 2011). The nucleic acid diversity among ANV isolates from different countries or from the same country indicates that these viruses are evolving rapidly (Mándoki et al., 2006).

In conclusion, ANV-1 was detected and partially characterised for the first time from Indian chicken flocks showing different clinical signs including diarrhoea, locomotion disorders, and stunted and uneven growth in addition to ne-

phritis. These findings warrant further investigations to identify the incidence of ANV in Indian poultry and its causative role in the above-mentioned diseases. The genetic diversity and economic impact of ANVs as well as the factors predisposing for the clinical or pathological manifestations also need to be explored in order to formulate suitable prevention and control measures.

\section{References}

Bosch, A., Guix, S., Krishna, N. K., Méndez, E., Monroe, E. E., Pantin-Jackwood, M. and SchultzCherry, S. (2012): Family Astroviridae. In: King, A. M. Q., Adams, M. J., Carstens, E. B. and Lefkowitz, E. (eds) Virus Taxonomy: Classification and Nomenclature of Viruses. Ninth Report of the International Committee on the Taxonomy of Viruses. Elsevier, pp. 953-959.

de Wit, J. J., Dam, G. B. T., van de Laar, J. M. A. M., Biermann, Y., Verstegen, I., Edens, F. and Schrier, C. C. (2011): Detection and characterization of a new astrovirus in chicken and turkeys with enteric and locomotion disorders. Avian Pathol. 40, 453-461.

Dufour-Zavala, L., Swayne, D. E., Glisson, J. R., Pearson, J. E., Reed, W. M., Jackwood, M. W. and Woolcock, P. (eds) (2008): A Laboratory Manual for the Isolation, Identification, and Characterization of Avian Pathogens. American Association of Avian Pathologists. 5th edition. Athens, GA, USA.

Hewson, K. A., O'Rourke, D. and Noormohammadi, A. H. (2010): Detection of avian nephritis virus in Australian chicken flocks. Avian Dis. 54, 990-993.

Imada, T., Taniguchi, T., Sato, S., Yamaguchi, S. and Kawamura, H. (1982): Pathogenicity of avian nephritis virus for embryonating hen's eggs. Natl Inst. Anim. Health Q. (Tokyo) 22, $8-15$.

Luna, L. (1968): Manual on Histologic Staining Methods of the Armed Force Institute of Pathology. 3rd edition. McGraw-Hill Book Co., New York, USA. pp. 32-46. 
Mándoki, M., Bakonyi, T., Ivanics, É., Nemes, C., Dobos-Kovács, M. and Rusvai, M. (2006): Phylogenetic diversity of avian nephritis virus in Hungarian chicken flocks. Avian Pathol. 35, 224-229.

Narita, M., Ohta, K., Kawamura, H., Shirai, J., Nakamura, K. and Abe, F. (1990): Pathogenesis of renal dysfunction in chicks experimentally induced by avian nephritis virus. Avian Pathol. J. 19, 571-582.

Ottiger, H.-P. (2010): Development, standardization and assessment of PCR systems for purity testing of avian viral vaccines. Biol. J. Int. Assoc. Biol. Stand. 38, 381-388.

Pantin-Jackwood, M. J., Strother, K. O., Mundt, E., Zsak, L., Day, J. M. and Spackman, E. (2011): Molecular characterization of avian astroviruses. Arch. Virol. 156, 235-244.

Shirai, J., Nakamura, K., Narita, M., Furuta, K., Hihara, H. and Kawamura, H. (1989): Visceral urate deposits in chicks inoculated with avian nephritis virus. Vet. Rec. 124, 658-661.

Shirai, J., Nakamura, K., Shinohara, K. and Kawamura, H. (1991): Pathogenicity and antigenicity of avian nephritis isolates. Avian Dis. 35, 49-54.

Shirai, J., Tanimura, N., Uramoto, K., Narita, M., Nakamura, K. and Kawamura, H. (1992): Pathologically and serologically different avian nephritis virus isolates implicated in etiology of baby chick nephropathy. Avian Dis. 36, 369-377.

Smyth, V. J., Jewhurst, H. L., Adair, B. M. and Todd, D. (2009): Detection of chicken astrovirus by reverse transcriptase-polymerase chain reaction. Avian Pathol. 38, 293-299.

Takase, K., Shinohara, K., Tsuneyoshi, M., Yamamoto, M. and Yamada, S. (1989): Isolation and characterisation of cytopathic avian enteroviruses from broiler chicks. Avian Pathol. 18, 631-642.

Tamura, K., Stecher, G., Peterson, D., Filipski, A. and Kumar, S. (2013): MEGA6: Molecular Evolutionary Genetics Analysis version 6.0. Mol. Biol. Evol. 30, 2725-2729.

Tang, Y., Ismail, M. M. and Saif, Y. M. (2005): Development of antigen-capture enzyme-linked immunosorbent assay and RT-PCR for detection of turkey astroviruses. Avian Dis. 49, $182-188$.

Todd, D., Trudgett, J., McNeilly, F., McBride, N., Donnelly, B., Smyth, V. J., Jewhurst, H. L. and Adair, B. M. (2010): Development and application of an RT-PCR test for detecting avian nephritis virus. Avian Pathol. 39, 207-213.

Todd, D., Trudgett, J., Smyth, V. J., Donnelly, B., McBride, N. and Welsh, M. D. (2011): Capsid protein sequence diversity of avian nephritis virus. Avian Pathol. 40, 249-259.

Yamaguchi, S., Imada, T. and Kawamura, H. (1979): Characterization of a picornavirus isolated from broiler chicks. Avian Dis. 23, 571-581. 